\title{
Searching for Light Boson via the Yukawa Process at Lepton Colliders
}

\section{Tanmoy Mondal* and Eung Jin Chun}

Korea Institute for Advanced Study, Seoul 02455, South Korea

E-mail: tanmoy@kias.re.kr, ejchun@kias.re.kr

I will present the prospect of Yukawa production process of a light boson which can exist in an extended Higgs sector. A particularly interesting case is the light pseudoscalar in Type-X two Higgs doublet model which can explain the anomalous magnetic moment of muon at large $\tan \beta$. Considering ILC "Higgs factory" with center-of-mass energy of $250 \mathrm{GeV}$, we show that the available parameter space can be fully examined by the (tau) Yukawa process at $5 \sigma$ with an integrated luminosity of $2000 \mathrm{fb}^{-1}$. We also demonstrate the mass reconstruction of such a light particle which helps to minimize the background events considerably. This proceeding is based on ref [1].

40th International Conference on High Energy physics - ICHEP2020

July 28 - August 6, 2020

Prague, Czech Republic (virtual meeting)

* Speaker 


\section{Introduction}

The two Higgs doublet model (2HDM) is one of the simplest extension of the Standard Model (SM) where two scalar doublets involve in the electroweak symmetry breaking (EWSB). Based on the Yukawa structure, there are four different types of 2HDM [2], and among them the Lepton-specific or Type-X 2HDM is of particular interest as it can explain the observation of muon anomalous magnetic moment $\left(\delta a_{\mu}\right)$ [3]. In the type-X scenario, a light pseudoscalar $(A)$ with mass $\left(m_{A}\right)$ less than the $Z$ boson mass with large $\tan \beta$ can explain the $\delta a_{\mu}$ anomaly, see [4] and references therein.

Although it is difficult to directly produce such light pseudoscalars at the LHC due to the hadrophobic nature, a light pseudoscalar can be searched readily at a lepton collider (LC) via the so-called Yukawa production process where a light $A$ is radiated from a tau lepton. There are several proposed lepton colliders (LC) like the international linear collider (ILC) for precision Higgs measurements. Any LC will run as Higgs factory where the center-of-mass energy $(\sqrt{s})$ will be $250 \mathrm{GeV}$ and it is worthwhile to study the prospect of light pseudoscalar search at a Higgs factory like ILC.

The Yukawa process is crucial to determine the pseudoscalar coupling to leptons independent of the other scalars which are present in the model. In this proceeding, we illustrate how to look for a light $A$ at a Higgs factory in the Yukawa process with four taus in the final state. It is possible to reconstruct the mass of the light pseudoscalar and we have demonstrated that by using the collinear approximation. We found that that it is possible to explore the whole $\delta a_{\mu}$ compatible parameter space of the Type-X $2 \mathrm{HDM}$ at the ILC with $2000 \mathrm{fb}^{-1}$ of integrated luminosity.

\section{The Type-X 2HDM Model}

For detail discussion about the model, please see ref [2]. The model consists of two scalar doublets $\Phi_{1}$ and $\Phi_{2}$ with hypercharge $Y=1$. The scalar potential is given by,

$$
\begin{aligned}
V_{2 \mathrm{HDM}}= & -m_{11}^{2} \Phi_{1}^{\dagger} \Phi_{1}-m_{22}^{2} \Phi_{2}^{\dagger} \Phi_{2}-\left[m_{12}^{2} \Phi_{1}^{\dagger} \Phi_{2}+\text { h.c. }\right]+\frac{1}{2} \lambda_{1}\left(\Phi_{1}^{\dagger} \Phi_{1}\right)^{2}+\frac{1}{2} \lambda_{2}\left(\Phi_{2}^{\dagger} \Phi_{2}\right)^{2} \\
& +\lambda_{3}\left(\Phi_{1}^{\dagger} \Phi_{1}\right)\left(\Phi_{2}^{\dagger} \Phi_{2}\right)+\lambda_{4}\left(\Phi_{1}^{\dagger} \Phi_{2}\right)\left(\Phi_{2}^{\dagger} \Phi_{1}\right)+\left\{\frac{1}{2} \lambda_{5}\left(\Phi_{1}^{\dagger} \Phi_{2}\right)^{2}+\text { h.c. }\right\} .
\end{aligned}
$$

Our results will not depend on nature of the couplings and for simplicity we have considered that all the couplings are real. After the electroweak symmetry breaking we express the doublets in the following way, $\Phi_{j}=\left(H_{j}^{+},\left(v_{j}+h_{j}+i A_{j}\right) / \sqrt{2}\right)^{T}$ and obtain the massive physical states $A$ (CP-odd), $h, H, H^{ \pm}$in terms of the gauge eigenstates:

$$
\left(\begin{array}{c}
H \\
h
\end{array}\right)=\left(\begin{array}{cc}
c_{\alpha} & s_{\alpha} \\
-s_{\alpha} & c_{\alpha}
\end{array}\right)\left(\begin{array}{l}
h_{1} \\
h_{2}
\end{array}\right), \quad A=-s_{\beta} A_{1}+c_{\beta} A_{2}, \quad \text { and } \quad H^{ \pm}=-s_{\beta} H_{1}^{ \pm}+c_{\beta} H_{2}^{ \pm} \text {. }
$$

Where $s_{\alpha}=\sin \alpha, c_{\beta}=\cos \beta$ etc and $\tan \beta=\frac{v_{2}}{v_{1}}$. We identify the CP-even state $h$ as the SM Higgs boson with mass $m_{h}=125 \mathrm{GeV}$. 


\begin{tabular}{|c||c|c|c|c|c|c|c|c|c|}
\hline & $\xi_{h}^{u}$ & $\xi_{h}^{d}$ & $\xi_{h}^{\ell}$ & $\xi_{H}^{u}$ & $\xi_{H}^{d}$ & $\xi_{H}^{\ell}$ & $\xi_{A}^{u}$ & $\xi_{A}^{d}$ & $\xi_{A}^{\ell}$ \\
\hline Type-X & $c_{\alpha} / s_{\beta}$ & $c_{\alpha} / s_{\beta}$ & $-s_{\alpha} / c_{\beta}$ & $s_{\alpha} / s_{\beta}$ & $s_{\alpha} / s_{\beta}$ & $c_{\alpha} / c_{\beta}$ & $\cot \beta$ & $-\cot \beta$ & $\tan \beta$ \\
\hline
\end{tabular}

Table 1: The multiplicative factors of Yukawa interactions in type X 2HDM

The Yukawa Lagrangian for Type-X 2HDM can be written in terms of mass eigenstates [5],

$$
\begin{aligned}
\mathcal{L}_{\text {Yukawa }}^{\text {Physial }=} & -\sum_{f=u, d, \ell} \frac{m_{f}}{v}\left(\xi_{h}^{f} \bar{f} h f+\xi_{H}^{f} \bar{f} H f-i \xi_{A}^{f} \bar{f} \gamma_{5} A f\right) \\
& -\left\{\frac{\sqrt{2} V_{u d}}{v} \bar{u}\left(\xi_{A}^{u} m_{u} P_{L}+\xi_{A}^{d} m_{d} P_{R}\right) H^{+} d+\frac{\sqrt{2} m_{l}}{v} \xi_{A}^{l} \bar{v}_{L} H^{+} l_{R}+\text { h.c. }\right\},
\end{aligned}
$$

where $u(d)$, and $\ell$ refer to the up (down)-type quarks, and charged leptons, respectively. The Yukawa multiplicative factors, i.e. $\xi_{\phi}^{f}$ are given in Table 1. In the limit $\cos (\beta-\alpha) \rightarrow 0$, the modifiers to the SM Higgs boson goes to +1 and matches with the SM Yukawa coupling. This is defined as right sign (RS) Yukawa limit. On the other hand, the lepton Yukawa modifier $\left(\xi_{h}^{\ell}\right)$ becomes ' -1 ' when $\cos (\beta-\alpha)$ takes the value $2 / \tan \beta$. This limit of $(\beta-\alpha)$ is known as wrong sign (WS) Yukawa limit.

\section{Search for Yukawa process at linear collider}

As discussed, we are interested in the Yukawa process: $e^{+} e^{-} \rightarrow Z^{*} / \gamma^{*} \rightarrow \tau^{+} \tau^{-} A \rightarrow 4 \tau$. Production cross section for this channel at the ILC for different values of $m_{A}$ is depicted in the left panel of Fig. 1 as a function of $\sqrt{s}$. Since the $A \tau \tau$ coupling is proportional to $\tan \beta$, cross-section increases as $\tan \beta$ increases. The tau leptons decay hadronically (65\%) or leptonically (35\%) and the signal events are identified as: $3 j_{\tau}+X$ where $X \equiv j_{\tau} / j / \ell_{\tau}$. Here $j_{\tau}$ is a $\tau$-tagged jet; $j$ is an untagged jet, and $\ell_{\tau}(\equiv e / \mu)$ is leptons from the decay of $\tau$. The total number of objects in a signal event is four. Since leptonic decay of a tau lepton is substantial, the inclusion of a lepton in the final state helps to maximize the signal event selection.

The dominant background comes from the $e^{+} e^{-} \rightarrow Z Z \rightarrow 4 \tau$ and $2 \tau 2 \mathrm{j}$ and processes where mis-identification of light jet into a $\tau$-tagged jet mimics the signal in the $2 \tau 2 j$ case. Subdominant background comes from the $e^{+} e^{-} \rightarrow Z h$ process. Parton level production cross-section of $4 \tau$ $(2 \tau 2 j)$ process is $6.6 \mathrm{fb}(225 \mathrm{fb})$ at $250 \mathrm{GeV}$ ILC with polarized beam.

\subsection{Event simulation and selection}

Parton level signal and background events are simulated with MadGraph5_aMC@NL0, PYTHIA8 and Delphes3 with the ILD detector card. Jets are clustered using the longitudinal-kT algorithm with $R=0.4$. In Delphes3, we assumed that the tau tagging efficiency of $\tau$ jets $\left(\epsilon_{\tau}\right)$ is 60\% [6] or $\epsilon_{\tau}=90 \%$ [7]. For both the cases we fix the mis-tagging rate at $0.5 \%$. To make opposite sign tau-jet pair, we used the Delphes jet charge measurement.

We imposed the pre-selection criteria that all the jets and leptons should have minimum energy of $20 \mathrm{GeV}$ and satisfy $|\cos \theta|<0.98$ which corresponds to $|\eta|<2.3$. Using the selected events we then move on to reconstruct the parent $\tau$-leptons. 

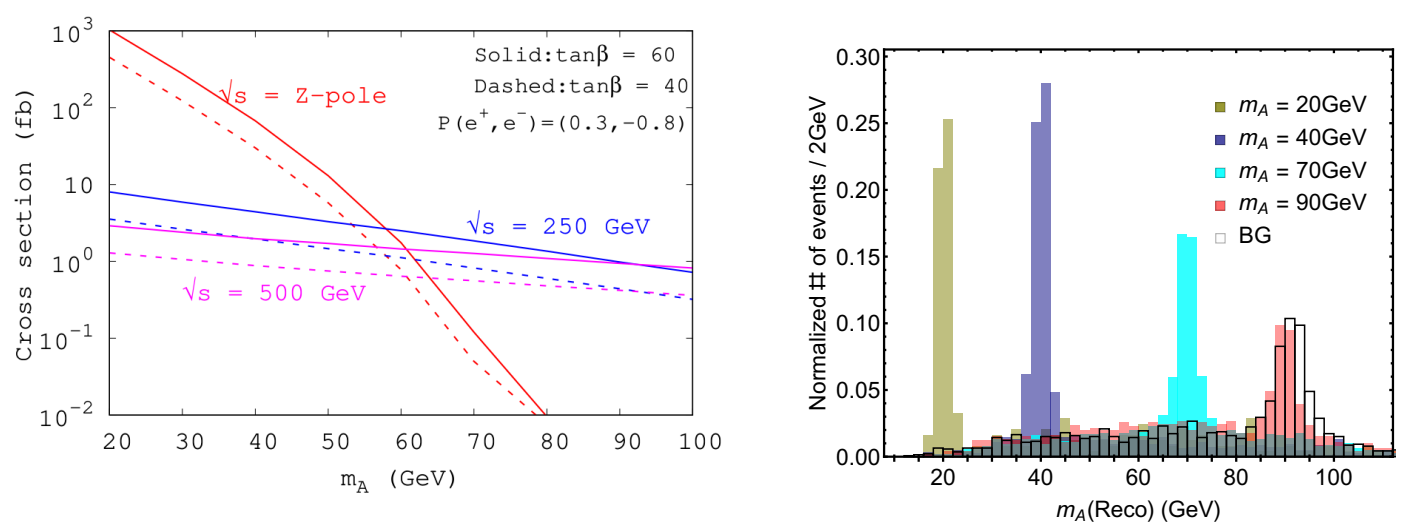

Figure 1: Left panel: Production cross section of $e^{+} e^{-} \rightarrow Z^{*} \gamma^{*} \rightarrow \tau \tau A$ final state for different A mass at different $\sqrt{s}$. Right panel: Normalized invariant mass distribution of the reconstructed pseudoscalar using collinear approximation for different pseudoscalar mass.

\subsection{Collinear approximation and reconstruction of $\mathrm{A}$}

At a lepton collider it is possible to reconstruct four taus by using the collinear approximation which assumes that the missing energy from the decay of tau lepton is collinear to the visible part of the decay. The energy momentum conservation equations are,

$$
\begin{aligned}
\vec{p}\left(\tau_{1}\right)+\vec{p}\left(\tau_{2}\right)+\vec{p}\left(\tau_{3}\right)+\vec{p}\left(\tau_{4}\right) & =\overrightarrow{0} \\
E\left(\tau_{1}\right)+E\left(\tau_{2}\right)+E\left(\tau_{3}\right)+E\left(\tau_{4}\right) & =\sqrt{s}
\end{aligned}
$$

We assume that the visible part of the tau decay carries $z_{i}$ amount of the original 4-momentum, i.e. $p^{\mu}\left(j_{i}\right)=z_{i} p^{\mu}\left(\tau_{i}\right)$ where $j_{i}$ is either a $\tau$-tagged jet or a light jet or a lepton. We then solve the above set of equations for $z_{i}$. The physical solutions should yield that $0<z_{i}<1$.

Since there are four $\tau \mathrm{s}$, there will be four possible candidates of opposite sign tau-pair $\left(\tau_{1}^{+} \tau_{1}^{-} / \tau_{2}^{+} \tau_{2}^{-} / \tau_{1}^{+} \tau_{2}^{-} / \tau_{2}^{+} \tau_{1}^{-}\right)$originate from the pseudoscalar. To identify the $A$ resonance without any ambiguity we use the following method :

- The highest energy $\tau$ out of the four is unlikely to come from the pseudoscalar. This is due to the fact that the maximum available energy for A varies from $126 \mathrm{GeV}\left(\right.$ for $\left.m_{A}=20 \mathrm{GeV}\right)$ to $141 \mathrm{GeV}\left(\right.$ for $\left.m_{A}=90 \mathrm{GeV}\right)$, whereas, energy of highest $\tau$ can reach close to $\sqrt{s} / 2$.

- From the remaining 3 taus one can construct two possible opposite sign tau-pair combinations. Among the two possible combinations we choose the $\tau$-pair which gives highest transverse momentum $\left(p_{T}\right)$ and the reconstructed invariant mass is defined as $m_{A}(\operatorname{Reco})$.

In the right panel of Fig. 1 we have plotted the $m_{A}$ (Reco) for different values of $m_{A}$ and the background events. The invariant mass peak becomes broader as $m_{A}$ increases, since the decay width of $A$ is proportional to its mass. Evidently, the method described above can be used for success full mass reconstruction out of the four taus. 

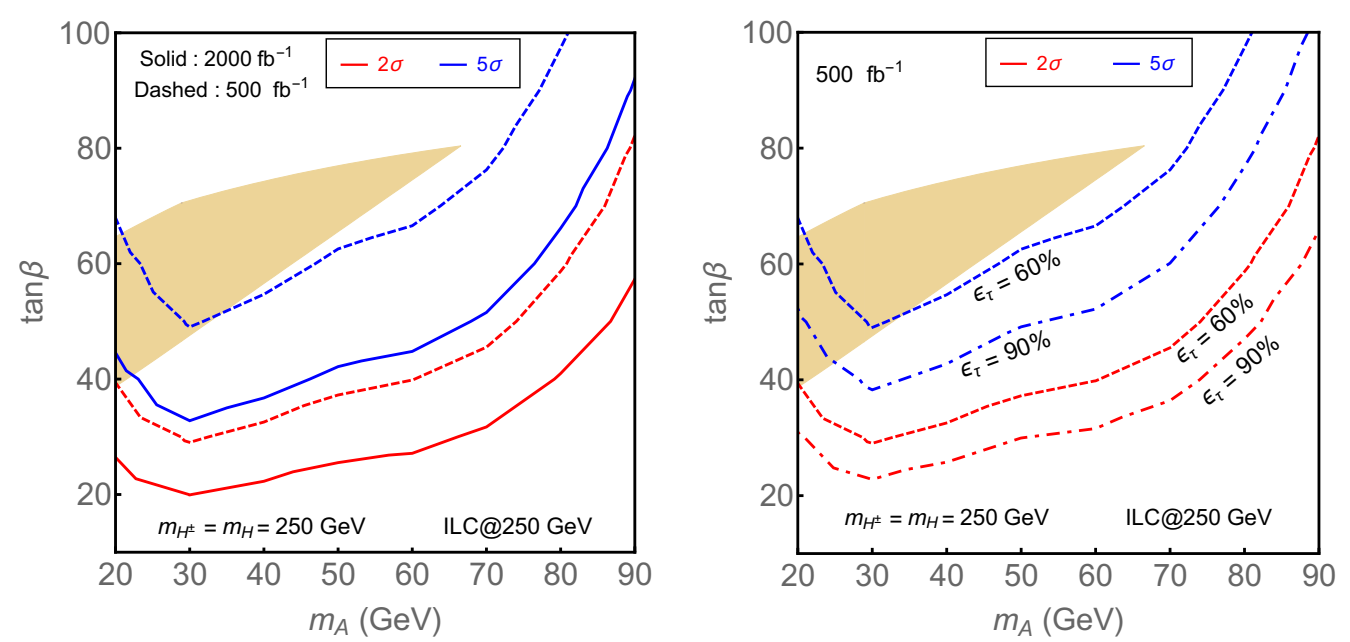

Figure 2: Left panel: Reach of the $250 \mathrm{GeV} e^{+} e^{-}$collider in the $m_{A^{-}} \tan \beta$ parameter space of the Type-X $2 \mathrm{HDM}$ with $\epsilon_{\tau}=60 \%$. The significance increases for larger $\tan \beta$ as the signal production cross section is almost proportional to $\tan ^{2} \beta$. The light yellow region can explain the $(g-2)_{\mu}$ anomaly at $2 \sigma$ after applying the lepton universality constraints. Right panel: Effect of better $\tau$-tagging efficiency is displayed here.

\section{Results}

In the previous section we have demonstrated that the collinear approximation can be used to reconstruct the mass and we use the reconstructed invariant mass to further minimize the background events. We define signal significance as, $\mathcal{S}=\sqrt{2\left[(S+B) \ln \left(1+\frac{S}{B}\right)-S\right]}$, where $S(B)$ are number of signal (background) events after the mass reconstruction cuts.

We scanned the $m_{A}-\tan \beta$ parameter space and computed the signal significance at ILC with $\sqrt{s}=250 \mathrm{GeV}$ with integrated luminosity of $500 \mathrm{fb}^{-1}$ and $2000 \mathrm{fb}^{-1}$. The $2 \sigma$ exclusion and $5 \sigma$ discovery contours are shown in the left panel of Fig. 2. The solid (dashed) contours corresponds to integrated luminosity of $2000(500) \mathrm{fb}^{-1}$ whereas the the red (blue) curves denote $2 \sigma(5 \sigma)$ significance. For the left panel plot we have used $\tau$-tagging efficiency of $60 \%$. As $m_{A}$ decreases the decay products become softer which leads to weak bound. At higher $m_{A}$, the signal cross-section decreases and as we move towards the $Z$-boson mass, it is difficult to distinguish the signal and background events. Consequently, the ILC reach becomes weak for higher $m_{A}$. The parameter space which can explain $\delta a_{\mu}$ after satisfying the lepton universality constraints coming from the tau decay and $Z \rightarrow \ell \ell$ measurements [8,9] is shown in dark yellow patch.

The tau-tagging efficiency at the ILC can be much better than LHC due to clean environment and in the right panel of Fig. 2 we have displayed the effect of better $\tau$-tagging efficiency. The dashed (dot-dashed) curves are for $\epsilon_{\tau}=60 \%(90 \%)$ and the red (blue) lines are for $2 \sigma(5 \sigma)$ significance. It is evident that a large portion of the parameter space which is favoured by the muon anomaly can be scrutinized at ILC@250 GeV with $500 \mathrm{fb}^{-1}$ luminosity where the exclusion limit goes below $\tan \beta=40$. With higher luminosity even more parameter space can be explored for this model. 


\section{Conclusion}

In Type-X 2HDM, a light pseudoscalar with large $\tan \beta$ can explain the observed excess of the muon anomalous magnetic moment and it is worthwhile to probe the scenario at high energy colliders like LHC or ILC. Due to the hadrophobic nature it is very hard to search the pseudoscalar at LHC unless the heavier Higgs bosons, $H^{ \pm}$and $H$, are lighter than about $200 \mathrm{GeV}$. Fortunately, lepton colliders are ideal to explore the relevant parameter space through the (tau) Yukawa process independent of the heavy Higgs masses.

We demonstrated that a Higgs-factory like the ILC at $250 \mathrm{GeV}$ can be useful to scrutinized the model regardless of the heavier Higgs masses. We perform a realistic analysis with the $4 \tau$ final states to reconstruct the light pseudoscalar by using the collinear approximation. The parameter space which can explain the muon $(g-2)$ anomaly can be explored at $5 \sigma$ with integrated luminosity of $2000 \mathrm{fb}^{-1}$.

\section{References}

[1] E. J. Chun and T. Mondal, Searching for a Light Higgs Boson via the Yukawa Process at Lepton Colliders, Phys. Lett. B 802 (2020) 135190 [1909. 09515].

[2] G. C. Branco, P. M. Ferreira, L. Lavoura, M. N. Rebelo, M. Sher and J. P. Silva, Theory and phenomenology of two-Higgs-doublet models, Phys. Rept. 516 (2012) 1 [1106.0034].

[3] Muon g-2 collaboration, Final Report of the Muon E821 Anomalous Magnetic Moment Measurement at BNL, Phys. Rev. D73 (2006) 072003 [hep-ex/0602035].

[4] L. Wang, J. M. Yang, M. Zhang and Y. Zhang, Revisiting lepton-specific 2HDM in light of muon g-2 anomaly, Phys. Lett. B788 (2019) 519 [1809. 05857].

[5] A. Broggio, E. J. Chun, M. Passera, K. M. Patel and S. K. Vempati, Limiting two-Higgs-doublet models, JHEP 11 (2014) 058 [1409. 3199].

[6] CMS Collaboration collaboration, Performance of reconstruction and identification of tau leptons in their decays to hadrons and tau neutrino in LHC Run-2, Tech. Rep.

CMS-PAS-TAU-16-002, CERN, Geneva, 2016.

[7] D. Jeans and G. W. Wilson, Measuring the CP state of tau lepton pairs from Higgs decay at the ILC, Phys. Rev. D98 (2018) 013007 [1804 . 01241].

[8] ALEPH, DELPHI, L3, OPAL, SLD, LEP Electroweak Working Group, SLD Electroweak Group, SLD Heavy Flavour Group collaboration, Precision electroweak measurements on the $Z$ resonance, Phys. Rept. 427 (2006) 257 [hep-ex/0509008].

[9] HFLAV collaboration, Averages of b-hadron, c-hadron, and $\tau$-lepton properties as of summer 2016, Eur. Phys. J. C77 (2017) 895 [1612.07233]. 